\title{
Research Note \\ Inter-Network magnetic fields observed during the minimum of the solar cycle
}

\begin{abstract}
J. Sánchez Almeida ${ }^{\star}$
Instituto de Astrofísica de Canarias, 38205 La Laguna, Tenerife, Spain

Received 26 June 2003 / Accepted 29 September 2003

Abstract. We analyze a time series of high angular resolution magnetograms of quiet Sun Inter-Network (IN) magnetic fields. These magnetograms have a spatial resolution better than 0.'5, a noise of some $20 \mathrm{G}$, and they have been obtained at the disk center during the minimum of the solar cycle. The IN regions show a typical unsigned flux density of the order of $15 \mathrm{G}$. Signals occur, preferentially, in the intergranular lanes, and the strongest signals trace a network with a scale similar to the mesogranulation. All these features are consistent with the IN magnetograms by Domínguez Cerdeña et al. (2003a,b), obtained during the maximum of the solar cycle. Consequently, the unsigned magnetic flux of the structures that give rise to the IN polarization signals does not seem to undergo large variations during the solar cycle.
\end{abstract}

Key words. Sun: granulation - Sun: magnetic fields - Sun: photosphere

\section{Rationale}

This note follows up the works by Domínguez Cerdeña et al. (2003a,b), where we analyzed high spatial resolution magnetograms of a quiet Sun Inter-Network (IN) region ${ }^{1}$. The IN magnetograms turned out to show much more unsigned magnetic flux than the values reported in the literature so far. In particular, the flux is larger than the unsigned flux in the form of active regions, which suggests the importance of the IN as far as the global magnetic properties of the Sun is concerned. (See the papers cited above and the references therein.)

Here I analyze a different series of quiet Sun magnetograms obtained by G. Scharmer ${ }^{2}$ during the developmental phases of a magnetograph for the Swedish Vacuum Solar Telescope (SVST, Scharmer et al. 1985; Scharmer 1989). They are simi-

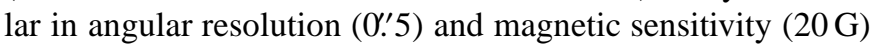
to those in Domínguez Cerdeña et al. (2003a,b) and, therefore, they offer an independent test for their results. Moreover, the SVST observations were obtained during the minimum

\footnotetext{
* e-mail: jos@ll.iac.es

1 The IN regions are those photospheric regions appearing as nonmagnetic in routine synoptic magnetograms. Magnetic signals in such regions were first reported by Livingston \& Harvey (1975) and Smithson (1975), and they appear in the literature with different names, e.g., inner network fields, intranetwork fields, granular fields, turbulent fields, etc.

${ }^{2}$ Quoted to be the series with higher angular resolution and polarimetric sensitivity obtained with the Swedish Vacuum Solar Telescope and this instrumentation; Scharmer (2003), private communication.
}

of the solar cycle (February 1996), as opposed to those of Domínguez Cerdeña et al. (2003a,b), gathered 6 years later (April 2002). This fortunate circumstance allows us to set constraints on the variation of the IN magnetic flux during the solar cycle.

The dataset and its calibration are discussed in Sect. 2. The results are put forward in Sect. 3. The implications of these results in the context of the previous IN measurements are discussed in Sect. 4.

\section{Observations and calibrations}

The quiet Sun region was observed on February 9, 1996, during the minimum of the solar cycle. A Field-Of-View (FOV) of $59^{\prime \prime} \times 81^{\prime \prime}$ includes several IN regions located very close to the solar disk center (see Fig. 1; the identification of the FOV within the Kitt Peak magnetogram was made by Stenflo \& Holzreuter 2003). The 0.'16 pixel size slighly undersamples the Airy disk of an ideal telescope with the diameter of the SVST $(\simeq 50 \mathrm{~cm})$ at the working wavelength $(\simeq 5250 \AA)$. The time series spans some $40 \mathrm{~min}$, with a 1 min cadence. Every snapshot of the series is made out of 44 images, each one with $210 \mathrm{~ms}$ exposure time. These images were selected as those of highest contrast during the $1 \mathrm{~min}$ interval of the cadence. The use of such frame selection technique contributes to the good spatial resolution of the resulting magnetograms. In addition, a tip-tilt mirror corrected for image motion during the individual exposures. The magnetograph consists of a nematic liquid crystal variable retarder, followed by a polarizing beam splitter and a 

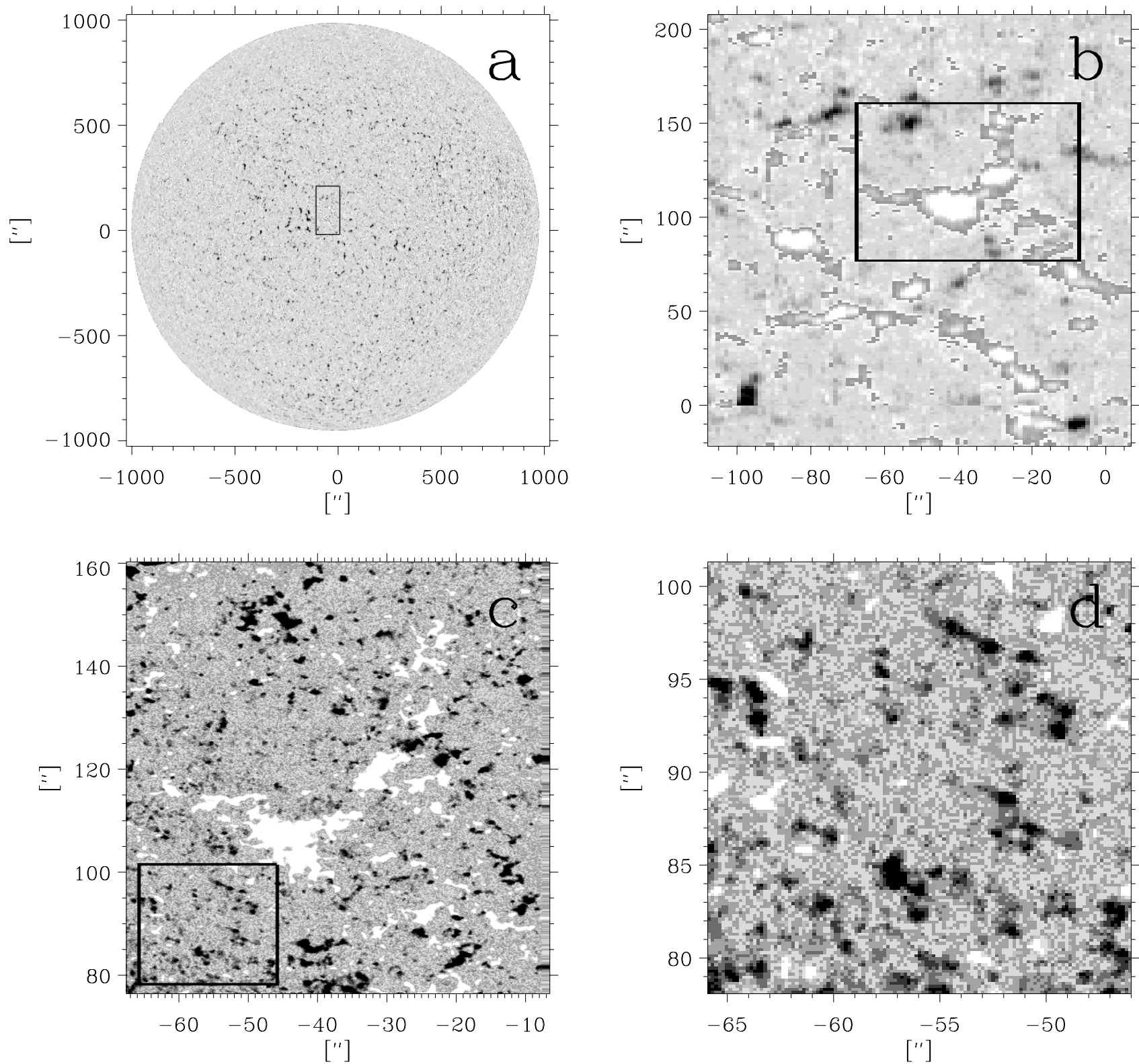

Fig. 1. Location of the SVST magnetograms on the solar surface. a) Kitt Peak full disk magnetogram of the same date obtained some $1 / 2$ hours later than the SVST magnetograms. b) Blowup of the rectangle in a). The new box indicates the position of the SVST magnetograms. c) SVST magnetogram. d) Blowup of the box in c). It corresponds to an IN region, as can be deduced by comparison with the magnetogram in b). All spatial scales are in arcsec refereed to the solar disk center. The scale of grays saturates at $\pm 80 \mathrm{G}$. Note that the aspect ratio of the different images is different.

narrow band Lyot filter (see also Zhang et al. 1998). The use of the beam splitter ameliorates the seeing induced instrumental polarization (see Lites 1987). The filter was tuned to select a wing of the magnetically sensitive line of Fe I at $5250.2 \AA$. It provides only a moderate spectral resolution $(150 \mathrm{~m} \AA$ at Full Width Half Maximum, FWHM). The central wavelength of the observation is unknown, since it was set by trial and error maximizing the polarization signals in some test magnetograms.

The magnetograms were provided uncalibrated, as a degree of circular polarization $V / I$ at the wavelength of observation. They have to be calibrated in units of flux densities $\left(\mathrm{Mx} \mathrm{cm}^{-2}\right)$ to allow comparison with other observations. (Since $1 \mathrm{Mx} \mathrm{cm}^{-2}=1 \mathrm{G}$, it is usual to employ $\mathrm{G}$ as the flux density unit, which is the convention adopted in this note.) Following the standard procedure, the calibration is based on the magnetograph equation which yields a linear relationship bewteen the longitudinal magnetic flux density $B_{\text {eff }}$ and the observed circular polarization (e.g., Unno 1956; Landi Degl'Innocenti 1992),

$B_{\text {eff }}=K V / I$.

Calibrating the magnetograms is therefore equivalent to determining the constant $K$, which is a non-trivial step since it depends on details of the observational setup (wavelength of observation, bandpass of the color filter, spectral line, thermodynamics of the atmosphere producing the polarization, etc.). 
We estimate the calibration constant in the quiet Sun, $K$ (quiet), from the signal $(V / I)_{0}$ observed in a pore existing in the FOV,

$K($ quiet $)=\left[B_{0} \frac{K \text { (quiet) }}{K(\text { pore })}\right] \frac{1}{(V / I)_{0}}$.

The flux density of the pore $B_{0}$ is assumed to be known,

$B_{0} \simeq 1650 \mathrm{G} \pm 250 \mathrm{G}$

with the error bar accounting for the range of values found in the literature (Sütterlin 1998, and references therein). The weakening factor in Eq. (2), $K$ (quiet) $/ K$ (pore), differs from one, and it has to be estimated. The line Fe I $\lambda 5250 \AA$ weakens with increasing temperature, and one expects a variation of the calibration constant between the atmospheres of a cold pore and the magnetic quiet Sun (e.g., Chapman \& Sheeley 1968; Harvey \& Livingston 1969). We estimate the weakening factor synthesizing Stokes $V$ profiles in various model atmospheres with different thermodynamic conditions but the same flux density. Then the ratio of calibration constants between two of such models is the inverse of the ratio of magnetograph signals (see Eq. (1)),

$\frac{K(1)}{K(2)}=\frac{(V / I)_{2}}{(V / I)_{1}}$.

The indexes 1 and 2 tag the two models. Using this equation and the dashed curve in Fig. 2, one finds

$\frac{K \text { (quiet) }}{K(\text { pore })} \simeq 1.6 \pm 0.4$

We have assumed the pore to be similar to a small sunspot, and the magnetic quiet Sun thermodynamics to lie in between the unmagnetized quiet Sun and the network. This range of possibilities provides the error bars given in Eq. (5). The other curves in Fig. 2 show that the weakening depends very little on the field strength of the model atmospheres (as far as the pore field strength is in the $\mathrm{kG}$ regime). Using Eqs. (3) and (5),

$B_{0} \frac{K \text { (quiet })}{K(\text { pore })} \simeq 2600 \mathrm{G} \pm 1100 \mathrm{G}$

which, together with Eqs. (1) and (2), render the final calibration,

$B_{\text {eff }}=(2600 \mathrm{G} \pm 1100 \mathrm{G}) \frac{V / I}{(V / I)_{0}}$.

The calibration procedure described above has the drawback of being model dependent. However, it automatically corrects for several potentially important systematic effects. First, the depolarization introduced by the SVST is believed to be large (e.g., the model Mueller matrix described by Sánchez Almeida et al. 1997 shows it to be as large as 50\%). The use of a ratio of signals, rather than $V / I$ alone, cancels it out ${ }^{3}$. Second, it accounts for the so-called saturation, since the fact that the

${ }^{3}$ One may also be concerned by the contamination of the Stokes $V$ signals with linear polarization signals. Although the SVST linear-to-circular polarization crosstalk is believed to be large, the solar linear polarization signals are very small (e.g., Sánchez Almeida \& Lites 2000), rendering a small contamination.

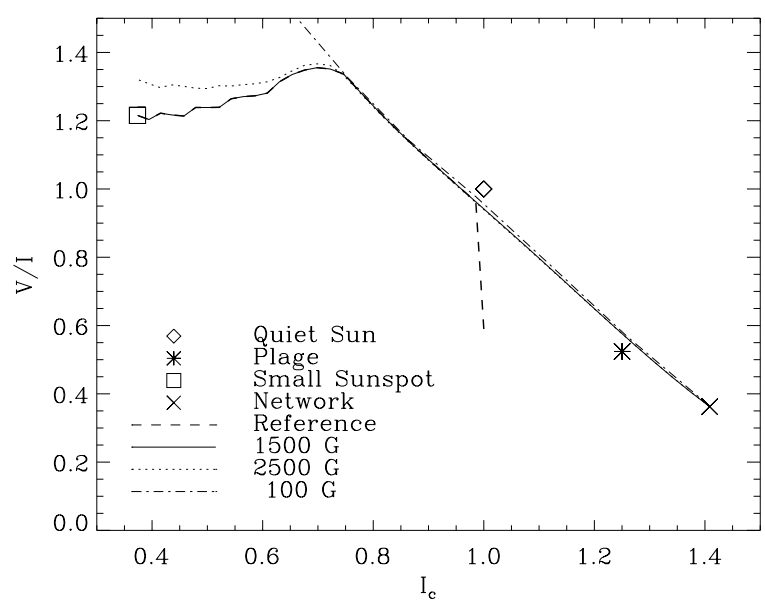

Fig. 2. Synthetic magnetograph signals $V / I$ for various model atmospheres with different thermodynamic properties, thus leading to different continuum intensity $I_{\mathrm{c}}$. The polarization and continuum intensities of Fe I $\lambda 5250 \AA$ are refereed to the signals in the quiet Sun model atmosphere by Maltby et al. (1986, the diamond symbol). The figure also includes synthetic signals for a model small sunspot (Collados et al. 1994, the box symbol), a model network (Solanki 1986, the times symbol), and a model plage (Solanki 1986, the asterisk). The magnetic field strength is constant with height and identical in all these four cases $(1500 \mathrm{G})$. The microturbulence has been set to $1 \mathrm{~km} \mathrm{~s}^{-1}$ whereas the color filter has been assumed to be Gaussian with a FWHM of $150 \mathrm{~m} \AA$. Following the observational procedure, the wavelength of observation has been chosen as that providing the largest $V$ signal. The solid line represents a set of model atmospheres whose thermodynamic conditions are linear combinations of the two most extreme model atmospheres (network and sunspot). The dotted line and the dotted-dashed line represent the same set of interpolated models except that the field strengths have been chosen to be $2500 \mathrm{G}$ and $100 \mathrm{G}$, respectively (see the inset). Finally, the dashed line shows the curve used for calibration. It is identical to the solid line, except that when the continuum intensity was larger than one, then $I$ from the quiet Sun synthesis was used to compute $V / I$. This artifice tries to account for the fact that the quiet Sun magnetic structures producing the observed signals are spatially unresolved (see, e.g., Domínguez Cerdeña et al. 2003a,b). Then the observed Stokes $I$ comes from a mean photosphere, rather than from the magnetic atmosphere.

Fe I $\lambda 5250 \AA$ polarization signals are not strictly proportional to the flux density for $\mathrm{kG}$ fields is taken into account by the syntheses. Finally, the calibration procedure allows to estimate the uncertainties involved in the calibration procedure, which will be used to assess the reliability of the conclusions.

The noise in the magnetograms was estimated from the difference between the signals of neighbor pixels, and from signals of the same pixel obtained in consecutive time steps. The noise of neighbor pixels is independent so, if the true signal in these pixels is assumed to be the same, then the difference only bears noise. Specifically, the standard deviation of the difference is $\sqrt{2}$ times the noise in a single pixel. We use the full series of magnetograms shifted in various directions to estimate the noise. All of them yield similar results; the noise turns out to be of the order of $20 \mathrm{G}$, a figure that we adopt for the noise level. Note that this value depends on the calibration of the magnetograms so it is $20 \pm 9 \mathrm{G}$, where the error bar just scales the uncertainty in Eq. (7). 


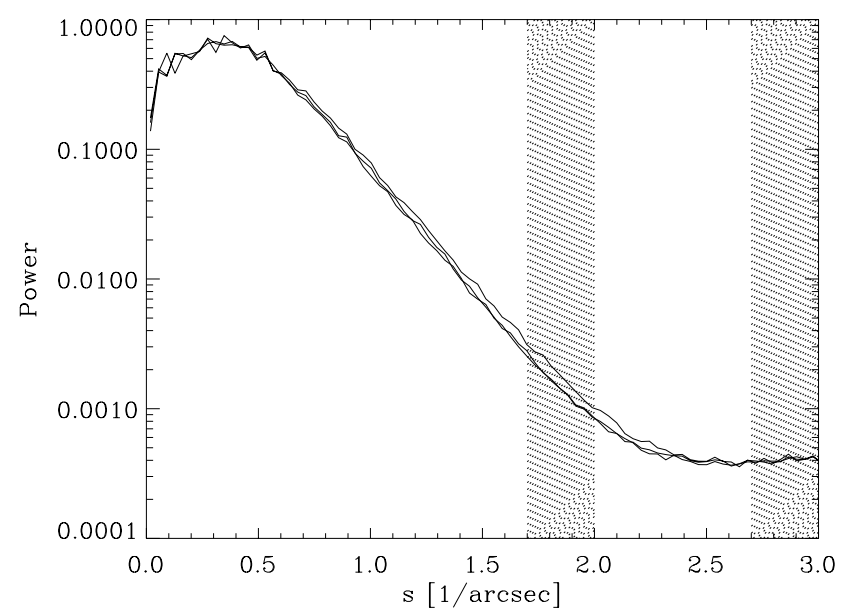

Fig. 3. Azimuthally integrated power spectra of the three best intensity images in the series. The spatial frequency, $s$, is given in $\operatorname{arcsec}^{-1}$. The images were selected as those whose power in the high-frequency signal bandpass $\left(1.7 \operatorname{arcsec}^{-1}<s<2 \operatorname{arcsec}^{-1}\right)$ is largest as refereed to the power in the noise bandpass $\left(2.7 \operatorname{arcsec}^{-1}<s<3 \operatorname{arcsec}^{-1}\right)$. (The hashed regions of the plot represent these bandpasses.) Note that signals exist up to frequencies larger than $2 \operatorname{arcsec}^{-1}$, which corresponds to a period of 0 .' 5 . The units of the power spectra have been arbitrarily chosen so that the maximum power is of the order of one.

In order to characterize the angular resolution of the observation, we rely on the power spectrum of the intensity images of the series. Figure 3 shows the azimuthally integrated power spectra of the three best images. They are the best images in the sense of having the largest power in a high frequency bandpass relative to the power in a noise bandpass (see the hashed regions in Fig. 3). It is clear that signals exist up to a frequency larger than $2 \operatorname{arcsec}^{-1}$, which corresponds to a period of $0 .{ }^{\prime} 5$. The contrast of these best images, i.e., the standard deviation over the mean value, is some $7 \%$. The best image in the series is chosen in the next section to quantify the amount of magnetic flux existing in the IN regions of the magnetograms. (All good snapshots provide similar fluxes.)

\section{Results}

Following Domínguez Cerdeña et al. (2003a,b), we characterize the amount of magnetic signals in the IN regions using the mean unsigned flux density, i.e., the mean value of the calibrated magnetogram once those signals below a threshold have been set to zero. (This quantity is defined in Eq. (16) of Domínguez Cerdeña et al. 2003b.) As it is worked out in the previous section, the noise in the magnetograms is of the order of $20 \mathrm{G}$. We use a threshold twice this value to minimize the contamination by noise. Three IN regions in the FOV were selected for analysis (see Fig. 4). The unsigned flux densities of the three regions are given in Table 1. All of them show similar values, which are of the order of $15 \mathrm{G}$. The table also includes the mean signed flux density (defined as the unsigned flux density but considering the sign of the signals), and the fraction of FOV covered by magnetic signals above noise. The same table contains the unsigned flux density of the magnetograms analyzed in Domínguez Cerdeña et al. (2003a,b) once the $40 \mathrm{G}$

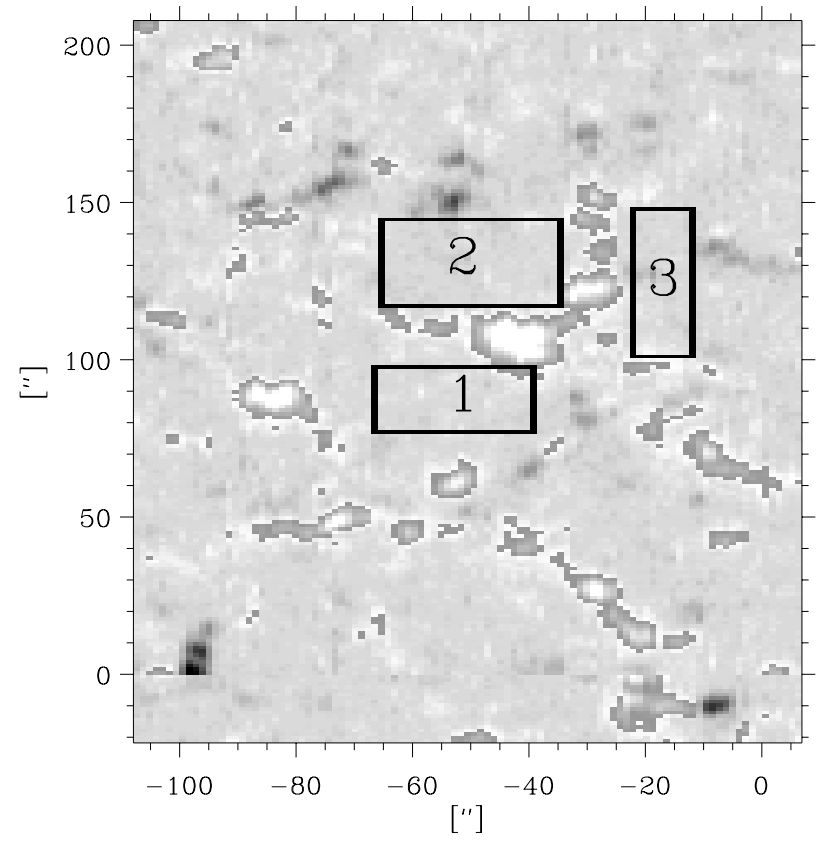

Fig. 4. Kitt peak magnetogram showing the three regions of the SVST magnetograms used for analysis. Note that they correspond to IN regions. The FOV is identical to Fig. 1b, but the signals are now scaled between $+150 \mathrm{G}$ and $-150 \mathrm{G}$ for clarify.

threshold used in this paper is taken into account. The unsigned flux is some $10 \mathrm{G}$ and therefore slightly smaller than the values that we obtain here. The same happens with the area covered by the signals, which is some $17 \%$ instead of the $25 \%$ of the SVST magnetograms. However, the uncertainty of the calibration can easily cope with the difference. Should the calibration constant be the lower limit in Eq. (6) $(1500 \mathrm{G})$, then the mean flux density would drop down to some $8 \mathrm{G}$.

Another clear result of the magnetograms of Domínguez Cerdeña et al. is the preference of the signals to appear on intergranular lanes. The same tendency is also found in the SVST magnetograms. Figure 5 shows the intensity image of IN region \# 1 overlaid with the corresponding magnetogram represented as contours. The magnetic signals show up on intergranular lanes, although not exclusively in there.

Yet another result of the works by Domínguez Cerdeña et al. is the existence of a web-like pattern with the spatial scale of the mesogranulation (say, between $5^{\prime \prime}$ and $10^{\prime \prime}$ ). This pattern is traced by the largest polarization signals. Although the pattern is not so clear in the magnetograms studied here, they contain small regions devoid of strong signals. Figure 6 contains two versions of the full FOV magnetogram showing signals within a certain range of flux densities. Figure 6a represents the strongest signals (larger than $150 \mathrm{G}$ ). It clearly depicts the supergranulation; the circle in the image has a diameter of 30", characteristic of the network pattern (e.g., Beckers 1981). Figure $6 \mathrm{~b}$ shows the strongest among the IN signals (signals whose absolute value ranges between $60 \mathrm{G}$ and $100 \mathrm{G}$ ). Voids with the mesogranular size are present (the circle on the image, with a diameter of $6^{\prime \prime}$, shows a scale typical of the 
Table 1. Mean flux densities in the magnetograms.

\begin{tabular}{ccccc}
\hline \hline & $\begin{array}{c}\text { Unsigned Flux } \\
{[\mathrm{G}]}\end{array}$ & $\begin{array}{c}\text { Signed Flux } \\
{[\mathrm{G}]}\end{array}$ & $\begin{array}{c}\text { Surface Coverage } \\
\%\end{array}$ & $\begin{array}{c}\text { Threshold }^{\mathrm{a}} \\
{[\mathrm{G}]}\end{array}$ \\
\hline IN Region \# 1 $^{\mathrm{b}}$ & $15 \pm 6$ & -5 & 28 & $40 \pm 17$ \\
IN Region \# 2 $^{\mathrm{b}}$ & $13 \pm 6$ & -1 & 22 & $40 \pm 17$ \\
IN Region \# 3 $^{\mathrm{b}}$ & $17 \pm 7$ & -3 & 26 & $40 \pm 17$ \\
full FOV & $30 \pm 13$ & 11 & 30 & $40 \pm 17$ \\
reference $^{\mathrm{c}}$ & 10 & & 17 & 40 \\
reference $^{\mathrm{c}}$ & 14 & & 28 & 30 \\
Kitt Peak full FOV $^{\mathrm{d}}$ & 9 & 8 & 8 & 40 \\
\hline
\end{tabular}

a The error bars account for the uncertainties in the calibration given in Eq. (7).

b Defined in Fig. 4.

c From the Fe I 6301 A magnetogram in Domínguez Cerdeña et al. (2003a,b).

d For the box shown in Fig. 1b.

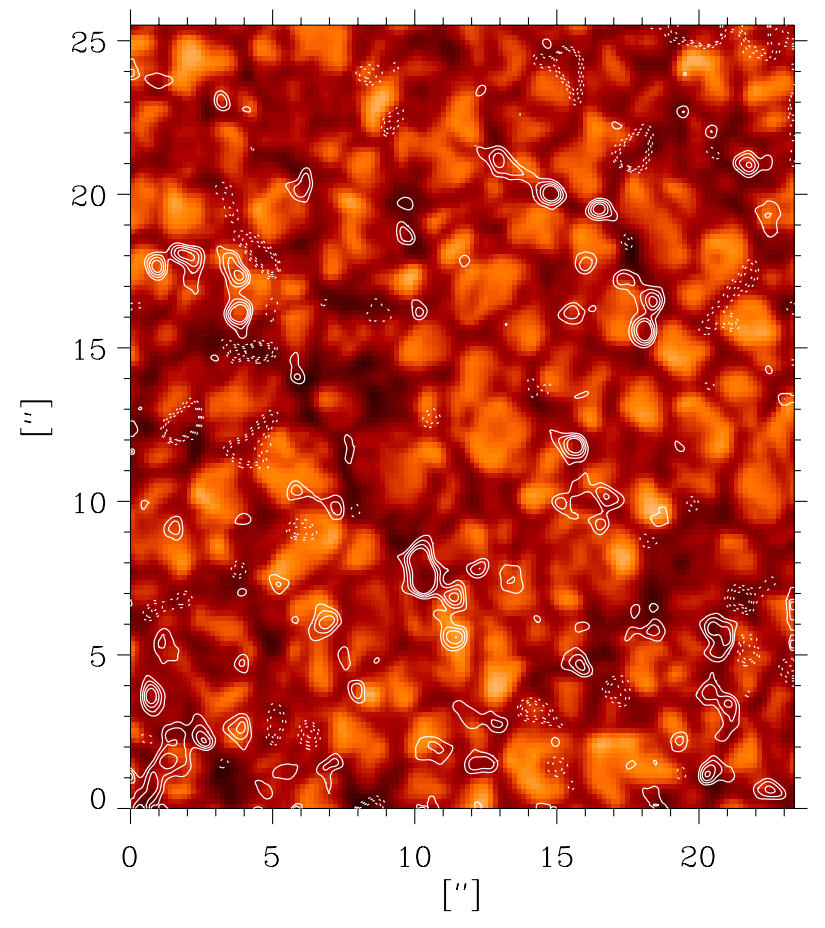

Fig. 5. Intensity image of the IN region \# 1, with the contours representing the magnetogram of the same region. Note how most of the signals are co-spatial with intergranular lanes, although this association is not one-to-one. The contours corresponds to $\pm 30 \mathrm{G}, \pm 50 \mathrm{G}$, $\pm 70 \mathrm{G}$ and $\pm 90 \mathrm{G}$, with the solid and dotted lines representing positive and negative polarities, respectively.

mesogranular pattern; see, e.g., November et al. 1981; Deubner 1989).

\section{Conclusions}

We estimate that the unsigned IN flux density at the disk center is of the order of $15 \pm 6 \mathrm{G}$. Some $25 \%$ of the IN regions is covered by signals above $40 \mathrm{G}$. (See Table 1 for a summary of results.) These figures for the unsigned flux density and area coverage are, within uncertainties, compatible with those obtained by Domínguez Cerdeña et al. (2003a,b) from magnetograms with similar angular resolution and a slightly better polarimetric sensitivity. The overall agreement has two main implications. First, this independent data set confirms the richness of IN magnetic features found by Domínguez Cerdeña et al. (2003a,b). Second, the SVST data used here was taken during the solar minimum, as opposed to the data by Domínguez Cerdeña et al. obtained at maximum. Since both datasets are consistent, we conclude that the IN flux density does not seem to vary along the cycle by more than $\pm 40 \%$ (i.e., within the error bars for the calibration of the present magnetograms). This narrow margin has to be compared with the signals of active regions. Active region flux at maximum is more than 10 times larger than the signals at minimum (see, e.g., Harvey-Angle 1993, Ch. 12, Fig. 9). The lack of IN flux variation suggests that active regions and IN fields have a different origin. In particular, it discards that the IN flux results from the decay of active regions, since it should be modulated according to the sunspot cycle ${ }^{4}$. These arguments are borrowed from Hagenaar et al. (2003), who use them to indicate the need of two uncoupled dynamos to produce active regions and network magnetic fields.

Obviously, the lack of variation along the cycle refers only to the kind of measurements that we analyze, i.e., 0.'5 angular resolution disk center observations of visible Zeeman signals above $40 \mathrm{G}$. For example, we have no information on the variation of the signals with latitude. Similarly, our result neither contradicts nor supports claims on the variation of the Hanle signals along the cycle (Faurobert et al. 2001). Hanle signals are tracing weak intrinsic field strengths which are probably not responsible for the polarization signals of visible spectral lines (see, e.g., Sánchez Almeida \& Lites 2000; Socas-Navarro \& Sánchez Almeida 2003).

\footnotetext{
${ }^{4}$ The same conclusion is reached from the strikingly different decay rates of active regions and IN fields; see Sánchez Almeida et al. (2003).
} 

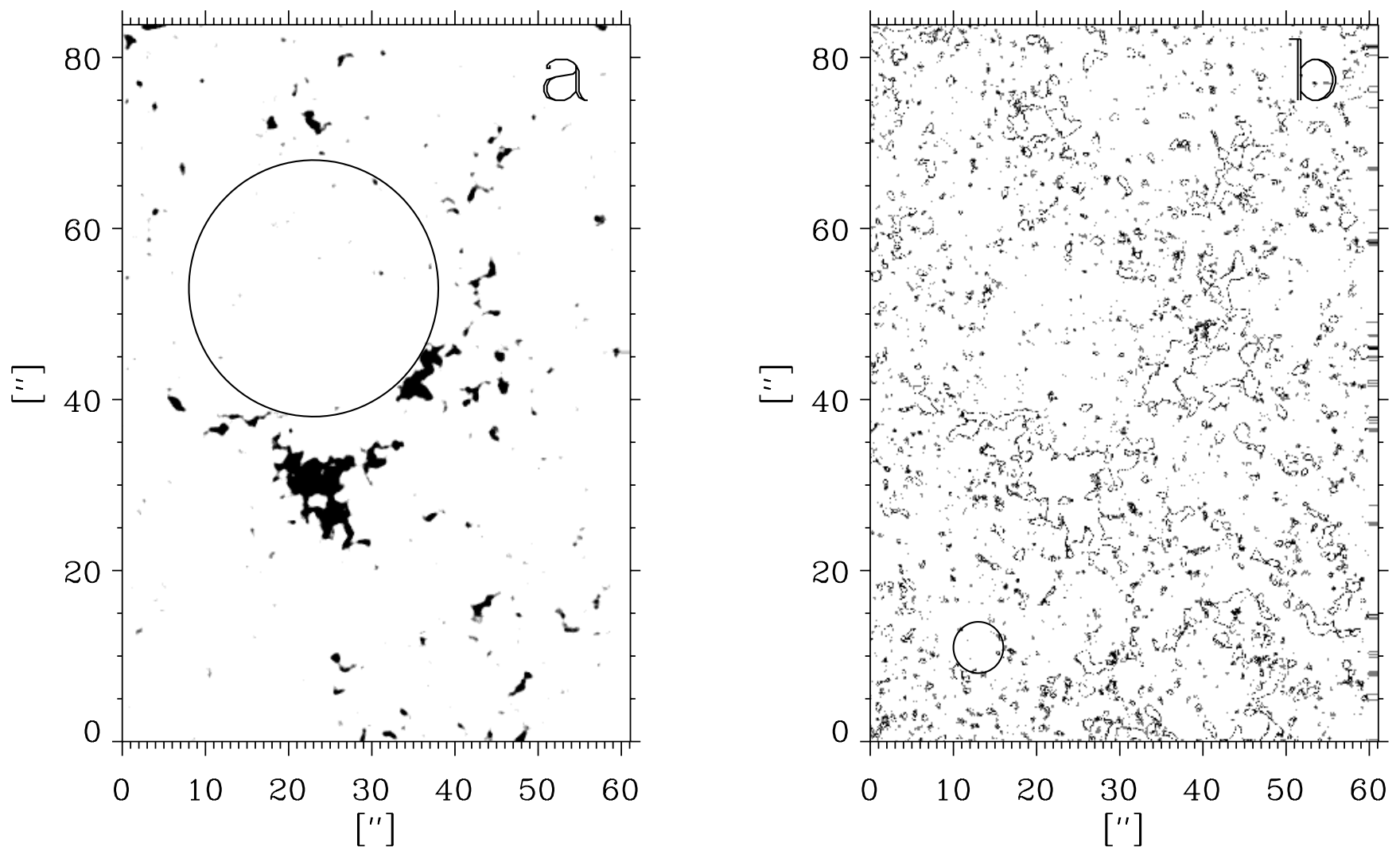

Fig. 6. Absolute value of the signals in the magnetogram for selected ranges of flux densities. a) Strongest signals in the magnetogram, i.e., those whose unsigned flux density is larger than $150 \mathrm{G}$. They trace the network. (The artificial circle, with a diameter of $30^{\prime \prime}$, has the typical size of a network cells.) b) Only strong IN signals are shown (flux densities between $60 \mathrm{G}$ and $100 \mathrm{G}$ ). The circle has a diameter of 6 ", typical of the mesogranular pattern.

Let us finish with a speculative detour. Stars with convective envelopes show emission in UV lines, which indicates the presence of hot chromospheres. Such emission has two components. One component is associated with the existence of magnetic fields, since it is well correlated with the parameters that characterize the efficiency of a global stellar dynamo. The second component, called basal flux, is always present independently of the stellar indexes tracing magnetic activity. According to the current paradigm, the origin of the basal flux is non-magnetic, being the residual heating due to dissipation of upward propagating waves. (For a full account of the current paradigm, see Schrijver 1995; see also Wunnenberg et al. 2002.) However, a magnetic field component whose properties remain constant along the cycle could also produce a residual chromospheric emission (see, e.g., Judge \& Carpenter 1998). The IN magnetic fields seems to fulfill this requirement and, therefore, they may contribute to the basal flux of the Sun and other solar-type stars.

Acknowledgements. The magnetograms were obtained by G. Scharmer with the SVST operated by the Royal Swedish Academy of Sciences in the Spanish Observatorio del Roque de los Muchachos. Thanks are due to the L. Rouppe van der Voort and D. Kiselman for help with the data handling. R. Rutten pointed out the relationship between the results in the note and the basal flux. Comments on the manuscript by I. Domínguez Cerdeña, H. Hagenaar, F. Kneer and G. Scharmer were very useful. The ISO/Kitt Peak data used here are produced cooperatively by NSF/NOAA, NASA/GSFC, and NOAA/SEL. The work was partly supported by the Spanish Ministerio de Ciencia y Tecnología, project AYA2001-1649.

\section{References}

Beckers, J. M. 1981, in The Sun as a Star, ed. S. Jordan, NASA SP-450 (Washington: NASA), 11

Chapman, G. A., \& Sheeley, N. R. 1968, Sol. Phys., 5, 442

Collados, M., Martínez Pillet, V., Ruiz Cobo, B., del Toro Iniesta, J. C., \& Vázquez, M. 1994, A\&A, 291, 622

Deubner, F. 1989, A\&A, 216, 259

Domínguez Cerdeña, I., Kneer, F., \& Sánchez Almeida, J. 2003a, ApJ, 582, L55

Domínguez Cerdeña, I., Sánchez Almeida, J., \& Kneer, F. 2003b, A\&A, 407, 741

Faurobert, M., Arnaud, J., Vigneau, J., \& Frish, H. 2001, A\&A, 378, 627

Hagenaar, H. J., Schrijver, C. J., \& Title, A. M. 2003, ApJ, 584, 1107 Harvey, J., \& Livingston, W. 1969, Sol. Phys., 10, 283

Harvey-Angle, K. L. 1993, Ph.D. Thesis, Utrecht University, Utrecht Judge, P. G., \& Carpenter, K. G. 1998, ApJ, 494, 828

Landi Degl'Innocenti, E. 1992, in Solar Observations: Techniques and Interpretation, ed. F. Sánchez, M. Collados, \& M. Vázquez (Cambridge: Cambridge University Press), 71

Lites, B. W. 1987, Appl. Opt., 26, 3838

Livingston, W. C., \& Harvey, J. W. 1975, BAAS, 7, 346

Maltby, P., Avrett, E. H., Carlsson, M., et al. 1986, ApJ, 306, 284

November, L. J., Toomre, J., Gebbie, K. B., \& Simon, G. W. 1981, ApJ, 245, L123 
Sütterlin, P. 1998, A\&A, 333, 305

Sánchez Almeida, J., Collados, M., Martínez Pillet, V., et al. 1997, in Advances in the Physics of Sunspots, ed. B. Schmieder, J. C. del Toro Iniesta, \& M. Vázquez, ASP Conf. Ser., 118, 366

Sánchez Almeida, J., Emonet, T., \& Cattaneo, F. 2003, in Solar Polarization 3, ed. J. Trujillo-Bueno, \& J. Sánchez Almeida (San Francisco: ASP), ASP Conf. Ser., 307, 293

Sánchez Almeida, J., \& Lites, B. W. 2000, ApJ, 532, 1215

Scharmer, G. B. 1989, in Solar and Stellar Granulation, ed. R. J. Rutten, \& G. Severino (Dordrecht: Kluwer), NATO ASI Ser., 263, 161

Scharmer, G. B., Brown, D. S., Pettersson, L., \& Rehn, J. 1985, Appl. Opt., 24, 2558
Schrijver, C. 1995, A\&AR, 6, 181

Smithson, R. C. 1975, BAAS, 7, 346

Socas-Navarro, H., \& Sánchez Almeida, J. 2003, ApJ, 593, 581

Solanki, S. K. 1986, A\&A, 168, 311

Stenflo, J. O., \& Holzreuter, R. 2003, in Current Theoretical Models and Future High Resolution Solar Observations: Preparing for ATST, ed. A. A. Pevtsov, \& H. Uitenbroek (San Francisco: Astronomical Society of the Pacific), ASP Conf. Ser., 286, 169

Unno, W. 1956, PASJ, 8, 108

Wunnenberg, M., Kneer, F., \& Hirzberger, J. 2002, A\&A, 395, L51

Zhang, H., Scharmer, G., Lofdahl, M., \& Yi, Z. 1998, Sol. Phys., 183, 283 